approach will allow a giant nonreciprocal response to be generated without applying an external magnetic bias.

\section{MEDICAL DIAGNOSTICS}

\section{Urine testing app}

Lab Chip 13, 4231-4238 (2013)

Monitoring the albumin levels in urine can be used to diagnose the early stages of kidney diseases, a growing global health problem. Because albumin levels fluctuate with time, tests should ideally be performed every few hours, but this demands successive patient visits to clinics. Now, Ahmet Coskun and colleagues from the University of California in Los Angeles, USA, have developed a lightweight, compact photonic attachment for a smart phone that can be used to measure albumin levels from urine samples. It uses a diode laser to simultaneously excite fluorescence in test and control samples. The fluorescence from both samples is then imaged using a lens and the existing camera unit on the smart phone. The tests take about $5 \mathrm{~min}$ to perform. Using buffer and synthetic urine samples, the researchers demonstrated that the platform has a detection limit of $5-10 \mu \mathrm{g} \mathrm{ml}^{-1}$, which is over three times better than the clinically accepted normal range of $\leq 30 \mu \mathrm{g} \mathrm{ml}^{-1}$. The researchers claim that the tester could be extended to enable simultaneous testing of multiple compounds, enhancing its usefulness for early detection of kidney-related diseases.

\section{LIQUID CRYSTALS}

\section{Nanosecond switching}

Phys. Rev. Lett. 111, 107802 (2013)

Because of their ability to change the polarization of light waves on application of an electric field, nematic liquid crystals form the basis of flat-screen displays in many kinds of consumer electronic devices. This polarization change typically occurs as a result of the electrically induced reorientation of molecules caused by the dielectric anisotropy of the material. However, this process, called the Frederiks effect, can be relatively slow with relaxation from the fieldon to the field-off state taking milliseconds. Now, Volodymyr Borshch and co-workers from Kent State University in the USA have reported a new electro-optic effect in liquid crystals that offers a switching time of the order of tens of nanoseconds or even nanoseconds. When a He-Ne laser beam with a wavelength of $633 \mathrm{~nm}$ and polarized at $45^{\circ}$ to the plane of incidence strikes a nematic liquid crystal (CCN-47) cell at $45^{\circ}$, the team observed that field-induced changes in birefringence follow both the 'on' and 'off' edges of the applied rectangular voltage pulses with a response time of about $30 \mathrm{~ns}$. According to them, the electro-optic effect is caused by the electric-field-induced modification of the order parameters (optic tensor) rather than Frederiks reorientation. The nanosecond switching ability of nematic liquid crystals may contribute to fundamental science and suggest new design options for displays. $R W$

\section{SUPERLUMINESCENT DIODES \\ High-optical power \\ Appl. Phys. Express 6, 092102 (2013)}

Superluminescent diodes are needed for applications that simultaneously require a high spatial coherence and a low temporal coherence, such as fibre-optic gyroscopes and optical coherence tomography. Now, Anna Kafar and colleagues from Poland and Japan have realized a blue-violet InGaN superluminescent diode that exhibits a high optical power of over $200 \mathrm{~mW}$ as a result of employing a bent-waveguide ('j-shaped') geometry. The design comprises two equal-length sections: one contains a straight waveguide, and the other has a curved waveguide whose axis is inclined at an angle relative to the output window. This arrangement has the advantage that light emitted towards the back facet travels twice the distance of light propagating towards the front facet, thereby enhancing light amplification and hence increasing the output power.

SP

\section{OPTICAL BEAMS \\ Vortex manipulation \\ Opt. Lett. 38, 3414-3417 (2013)}

Optical vortices - light beams with twisted wavefronts - are employed in numerous applications, including quantum entanglement and communications. Research into vortex beams is now focusing on how to convert and manipulate their wavefront topology. Now, Yaroslav Kartashov and co-workers from Spain and Russia have numerically demonstrated that spiralling shallow refractive index landscapes induce coupling and periodic energy exchange between different topological light states. The scientists considered resonant excitation of vortex modes in a waveguide that has a helical refractive index profile. They investigated propagation of light beams with a wavelength of $633 \mathrm{~nm}$ in fused silica with a refractive index contrast of $7 \times 10^{-4}$. Numerical simulations of the intensity and phase distributions revealed an evolution from the $m=0$ mode to the $m=1$ mode, where $m$ is the topological charge. The energy exchange between these two modes varied sinusoidally as a function of the propagation distance, whereas its period was determined by the difference of the propagation constants of the two modes.

\section{OPTICAL MANIPULATION} Metasurface spin effect Nano Lett. 13, 4148-4151 (2013)



The transport of angular momentum of light occurs in two components: the orbital and spin angular momenta. The interaction between the orbital and spin angular momenta can be put to good use; for example, it can be used in optical communications and information processing applications. Now, using metasurfaces - a class of structured interfaces with varying profiles of nanostructures Guixin Li and colleagues from Hong Kong and the UK have investigated the interaction between the orbital and spin angular momenta of light. The metasurfaces are constructed from geometric phase elements and designed to have 60 concentric circular rings featuring Z-shaped apertures etched on a gold surface. The rings have a periodicity of $300 \mathrm{~nm}$, which is less than half the incident wavelength of $750 \mathrm{~nm}$. The spin-orbit interaction between the incident light and the metasurfaces originates from the geometric orientations of the metamaterial atoms. When light with linear polarization in the $y$-direction (equivalent to the superposition of two circular polarizations) was incident on the metasurfaces, the researchers measured spininduced angular splitting between two circular polarizations as a result of the optical spin Hall effect. They also showed that the phase shifts between orbitals could be controlled through the metasurfaces to induce a global orbital rotation by carrying the spin effects to orbitals. These findings will aid the understanding and design of spin-enabled metasurfaces for manipulating optical vortex beams.

Written by Oliver Graydon, Noriaki Horiuchi, David Pile, Simon Pleasants and Rachel Won 